\title{
Comparison of rose bengal plate test, serum agglutination test, and indirect enzyme-linked immunosorbent assay in brucellosis detection for human and goat samples
}

Ipola Patrick Albert ${ }^{1,2}$, Charles Drago Kato ${ }^{2}$, Kokas Ikwap ${ }^{2}$, Steven Kakooza ${ }^{3}$, Benson Ngolobe ${ }^{2}$, Dickson Ndoboli ${ }^{1}$ and Gabriel Tumwine ${ }^{1}$

1. Uganda Research Training Collaborative, Kampala, Uganda; 2. Department of Biomolecular Resources and Biolab Sciences College of Veterinary Medicine Animal Resources and Biosecurity, Makerere University, P. O. Box 7062 Kampala, Uganda; 3. Central Diagnostic Laboratory, College of Veterinary Medicine Animal Resources and Biosecurity, Makerere University, P. O. Box 7062 Kampala, Uganda; Novel Minds Science Plexus, Uganda.

Corresponding author: Ipola Patrick Albert, e-mail: patrickalbert4@outlook.com Co-authors: CDK: katodrago@yahoo.com, KI: kikwap@gmail.com, SK: ksteven310gmail.com, BN: ngolobe.ben19@gmail.com, DN: dickson.ndoboli@gmail.com, GT: tumwinegabriel@gmail.com Received: 23-02-2018, Accepted: 18-06-2018, Published online: 11-07-2018

doi: 10.14202/IJOH.2018.35-39 How to cite this article: Ipola PA, Kato CD, Ikwap K, Kakooza S, Ngolobe B, Ndoboli D, Tumwine G. Comparison of rose bengal plate test, serum agglutination test, and indirect enzyme-linked immunosorbent assay in brucellosis detection for human and goat samples. Int J One Health 2018;4:35-39.

\begin{abstract}
Background: In Uganda, detection of brucellosis in goats and humans is more frequently done using rose bengal plate test (RBPT) in comparison to other serological tests such as serum agglutination test (SAT) and indirect enzyme-linked immunosorbent assay (i-ELISA).
\end{abstract}

Aim: This study aimed at estimating the degree of agreement, specificity and sensitivity of RBPT, SAT, and ELISA in brucellosis detection in human and goat samples.

Materials and Methods: An analytical study was conducted involving parallel testing of 425 goat samples and 177 human samples to compare RBPT, SAT, and i-ELISA tests of brucellosis detection.

Results: A seroprevalence of $14.82 \%$ in animals and $11.86 \%$ in humans was detected using RBPT, $7.06 \%$ in goats and $5.09 \%$ in humans using SAT, and $6.35 \%$ in humans and $4.52 \%$ in animals using i-ELISA. The degree of agreement between the three tests in humans and animals was compared using Fleiss' kappa statistics where a moderate agreement (MA) was obtained for both with kappa value 0.57 in animals and 0.51 in humans and $p<0.001$. Kappa values in animals were 0.52 (MA) for RBPT and i-ELISA, 0.61 (MA) for RBPT and SAT, and 0.82 substantial agreement (SA) for SAT and i-ELISA. The kappa values in humans were 0.52 (MA) for RBPT and i-ELISA, 0.57 (MA) for RBPT and SAT, and 0.82 (SA) for SAT and i-ELISA, respectively. The study revealed a significant agreement in the tests for all the three tests, but there was SA between SAT and i-ELISA in both animal and human samples. Sensitivity and specificity of RBT and SAT were estimated using i-ELISA as a gold standard as follows, in goats RBPT: 100 and 91\% and SAT: 100 and 99\%, respectively, and in humans RBPT: 100 and 92\% and SAT: 88 and $99 \%$, respectively.

Conclusion: It appears that RBPT is good as a screening test, whereas SAT and i-ELISA are good for diagnosis purposes or confirmatory test.

Keywords: brucellosis, indirect enzyme-linked immunosorbent assay, rose bengal plate test, serum agglutination test, Uganda.

\section{Introduction}

Brucellosis is one of the most common neglected zoonotic diseases, and it has continued to widely affect the world's population with up to 500,000 new cases worldwide annually in humans, greatly affecting the developing countries such as India, Uganda, Sudan, and among others [1-3]. A study conducted in Kampala city in 2011 found out that adjusted herd prevalence of brucellosis was $6.5 \%(11 / 177,95 \%$

Copyright: Albert, et al. This article is an open access article distributed under the terms of the Creative Commons Attribution 4.0 International License (http://creativecommons.org/licenses/ by/4.0/), which permits unrestricted use, distribution, and reproduction in any medium, provided you give appropriate credit to the original author(s) and the source, provide a link to the Creative Commons license, and indicate if changes were made. The Creative Commons Public Domain Dedication waiver (http:// creativecommons.org/ publicdomain/zero/1.0/) applies to the data made available in this article, unless otherwise stated. confidence interval [CI]: 3.6-10.0\%) and the adjusted individual animal prevalence was 5.0\% (21/423, 95\% CI: $2.7-9.3 \%$ ) based on diagnosis using commercial kits of the competitive enzyme-linked immunosorbent assay (ELISA) for Brucella abortus antibodies [4].

Several methods are used to detect and screen brucellosis that includes rose bengal plate test (RBPT), ELISA, serum agglutination test (SAT), complement fixation test, indirect hemolysis test, and among others [5]. Some of the tests used for brucellosis are highly specific or highly sensitive, thus leading to wrong/misdiagnosis of brucellosis in humans and the animals [6]. Misdiagnosis and underreporting are because in many cases patients have pyrexia of unknown origin or the symptoms and signs are confused with those of other diseases such as typhoid fever, rheumatic fever, spinal tuberculosis, pyelitis, 
cholecystitis, thrombophlebitis, autoimmune disease, and tumors [7]. The most common test that is used to detect and screen brucellosis in sub-Saharan Africa, which includes countries like Uganda is RBPT due to its cheap cost [8].

This study aimed at estimating the degree of agreement, specificity and sensitivity of RBPT, SAT, and ELISA in brucellosis detection in human and goat samples.

\section{Materials and Methods \\ Ethical approval}

The research methods were reviewed and approved by the Research and Ethics Committee of the School of Biosecurity, Biotechnical and Laboratory Sciences (SBLS) approval number SBLS/REC/16/17, and School of Veterinary Medicine and Animal Resources, College of Veterinary Medicine, Animal Resources and Biosecurity, Makerere University.

\section{Study area}

The research was done in Uganda, and the samples were obtained from samples submitted to Central Diagnostic Laboratory (CDL) for brucellosis screening and those from other studies for brucellosis. CDL located at College of Veterinary Medicine Animal Resources and Biosecurity, Makerere University, was used for the laboratory procedures.

\section{Study design}

An analytical study was conducted involving analytical methods of data collection, which included the use of i-ELISA, SAT, and RBT techniques of sample analysis and then data collected was entered into Excel and analyzed using SPSS version 22. Parallel testing of the samples was done where each sample was subjected to all the three tests, SAT, RBT, and ELISA.

\section{Sample collection and handling}

A total of 425 goat samples collected from Serere district and 177 human samples collected from Nakasongola between January and April 2017 were randomly chosen from the batch of archived samples, at CDL. All the samples achieved for animals had either aborted or reproductive disorders like infertility. All human samples were suspected for brucellosis based on clinical signs like headache, fever, joint muscle and back pain, fatigue, sweats, loss of appetite, body weakness among others.

\section{Procedure of RBPT}

RBPT was performed according to standard procedure [9] with little modifications. $75 \mu \mathrm{L}$ of serum was taken on a rose bengal plate by micropipette. The rose bengal-colored antigen bottle was shaken well to ensure homogenous suspension, and then $25 \mu \mathrm{L}$ of the rose bengal-colored antigen was added to the serum. The antigen and serum were mixed thoroughly and waited for $4 \mathrm{~min}$. The result was observed immediately after $4 \mathrm{~min}$. Definite clumping/agglutination was considered as a positive reaction, whereas no clumping/agglutination was regarded as a negative. Controls were run before the sample tests were done.

\section{Procedure for SAT}

SAT was performed in 2-fold dilution using phenol as a diluent. Briefly, $0.8,0.5,0.5,0.5$, and $0.5 \mathrm{~mL}$ of $0.5 \%$ diluent (phenol) were dispensed to tube 1,2 , 3,4 , and 5 , respectively. $0.2 \mathrm{~mL}$ of sample serum was dispensed to tube 1 , and the same was done with the positive and negative control. The diluent and serum were mixed well, and 2-fold dilution was done, starting by pipetting of $0.5 \mathrm{~mL}$ from tube 1 , such that serum dilution of $1 / 5,1 / 10,1 / 20,1 / 40$, and $1 / 80$ was obtained in tube $1,2,3,4$, and 5 , respectively. $0.5 \mathrm{~mL}$ of $1 / 10$ diluted antigen with $10 \%$ phenol was dispensed to all tubes and mix well and incubated at $37^{\circ} \mathrm{C}$ for $16-20 \mathrm{~h}$. The final serum dilution was $1 / 10,1 / 20$, $1 / 40,1 / 80$, and $1 / 160$ in tubes $1,2,3,4$, and 5 , respectively. A control panel for reading of results was made. Units were converted to international units per $\mathrm{mL}$. Serum that agglutinated in a tube containing $30 \mathrm{IU} /$ $\mathrm{mL}$ or more was considered positive, and those that had lower than $30 \mathrm{IU} / \mathrm{mL}$ were considered negative.

\section{Procedure for i-ELISA}

i-ELISA was performed using commercially available kits according to the manufacturer's protocol (IDEXX Montpellier SAS, 326 rue de la Galera, 34090 Montpellier, FR 04-55011-00).

The data obtained were entered into Excel spreadsheets and then transferred to STATA and SPSS version 16 for analysis.

\section{Results}

Seroprevalence of brucellosis in animals (goats) by RBPT, SAT, and i-ELISA tests

A total of 425 animal samples (goats) were parallelly analyzed using SAT, i-ELISA, and RBPT. Of the 425 samples, RBPT revealed a prevalence of $14.82 \%$, $7.06 \%$ with SAT, and $6.35 \%$ with IDEX i-ELISA. All the samples positive with i-ELISA were positive with SAT and RBPT. The results are shown in Table-1.

\section{Agreement between SAT, i-ELISA, and RBPT for ani- mal samples}

An inter-rater reliability test was performed to analyze the degree of agreement between RBPT, SAT, and i-ELISA using Fleiss' kappa. The inter-rater reliability for RBPT, SAT, and i-ELISA in animal samples found moderate agreement (MA) with Kappa $=0.57(\mathrm{p}<0.001)$, $95 \%$ CI which was statistically significant. Cohen's kappa was done to establish the degree of agreement between two tests. The results are shown in Table-2.

\section{Seroprevalence of brucellosis in human samples by RBPT, SAT, and i-ELISA tests}

A total of 177 human samples were parallelly analyzed using SAT, RBPT, and i-ELISA. Of the 177 samples, RBPT revealed a prevalence of 11.86 , $5.09 \%$ with SAT, and $4.52 \%$ with i-ELISA. One case was positive for RBPT, negative for SAT, but positive with i-ELISA. The results are shown in Table-3. 
Agreement between RBPT, SAT, and i-ELISA for human samples

An inter-rater reliability test was performed to analyze the degree of agreement between RBPT, SAT, and i-ELISA using Fleiss' kappa. The inter-rater reliability for RBPT, SAT, and i-ELISA in human samples found MA with Kappa=0.51 $(\mathrm{p}<0.001), 95 \% \mathrm{CI}$ which was statistically significant. The results are shown in Table-4.

Sensitivity and specificity of RBPT and SAT by comparing with i-ELISA as a gold standard for detection of brucellosis antibodies in goat and human samples

The sensitivity and specificity of RBPT and SAT were estimated using i-ELISA as a gold standard. RBPT had the highest sensitivity in both human and goat samples, whereas SAT had the highest specificity in both human and goat samples. The results are shown in Table-5.

\section{Discussion}

In Uganda, different tests have been used in the detection of brucellosis in the field and hospitals. Some of the tests used for brucellosis detection are highly specific or highly sensitive, thus leading to wrong/misdiagnosis of brucellosis in humans and the animals [6]. There are several serological tests that are used for brucellosis detection that includes RBPT, SAT, and i-ELISA, among others, i-ELISA is regarded as the most specific test for brucellosis detection and RBPT most sensitive test [10]. This study revealed similar results; however, there was MA when all the three tests were compared for both human and animal samples. The degree of agreement for all the tests for human and animal samples was statistically significant with $p<0.001$ and kappa statistic of 0.57 for animal samples and 0.51 for human samples.

Rose bengal test had the highest seroprevalence in both animal and human samples $14.82 \%$ in animals and $11.86 \%$ in humans similar to results observed by Din [11], whereas results by Bhanderi showed i-ELISA with the highest seroprevalence [12]. The high seroprevalence in RBPT could be due to false-positive results with RBPT. These results may occur for a variety of many reasons which are found most prominent due to cross-reactions with other bacteria including Yersinia enterocolitica 0:9, which share with Brucella spp. a major O-polysaccharide almost completely, also serological cross-reactions between

Table-1: Seroprevalence of brucellosis in animals (goats) by RBPT, SAT, and i-ELISA tests.

\begin{tabular}{lccccc}
\hline Test & $\begin{array}{c}\text { Number of } \\
\text { samples tested }\end{array}$ & $\begin{array}{c}\text { Number of } \\
\text { positives }\end{array}$ & $\begin{array}{c}\text { Number of } \\
\text { negatives }\end{array}$ & $\begin{array}{c}\text { Prevalence by } \\
\text { each test (\%) }\end{array}$ & $\begin{array}{c}\text { Number of positives with RBPT negative with } \\
\text { SAT but positive with ELISA }\end{array}$ \\
\hline RBPT & 425 & 63 & 362 & 14.82 & 0 \\
SAT & 425 & 30 & 395 & 7.06 & 0 \\
i-ELISA & 425 & 27 & 398 & 6.35 & 0 \\
\hline
\end{tabular}

i-ELISA: Indirect enzyme-linked immunosorbent assay, SAT: Serum agglutination test, RBPT: Rose bengal plate test

Table-2: The degree of agreement between RBPT, SAT, and i-ELISA tests for animal samples.

\begin{tabular}{lcccl}
\hline Tests & Kappa & p value & CI (\%) & Degree of agreement \\
\hline RBPT and SAT & 0.61 & $<0.001$ & 95 & Substantial agreement \\
RBPT and i-ELISA & 0.52 & $<0.001$ & 95 & Moderate agreement \\
SAT and i-ELISA & 0.82 & $<0.001$ & 95 & Substantial agreement \\
RBPT, SAT, and i-ELISA & 0.57 & $<0.001$ & 95 & Moderate agreement \\
\hline
\end{tabular}

i-ELISA: Indirect enzyme-linked immunosorbent assay, SAT: Serum agglutination test, RBPT: Rose bengal plate test, $\mathrm{CI}$ : Confidence interval

Table-3: Seroprevalence of brucellosis in human samples by RBPT, SAT, and i-ELISA tests.

\begin{tabular}{lccccc}
\hline Test & $\begin{array}{c}\text { Number of } \\
\text { samples tested }\end{array}$ & $\begin{array}{c}\text { Number of } \\
\text { positives }\end{array}$ & $\begin{array}{c}\text { Number of } \\
\text { negatives }\end{array}$ & $\begin{array}{c}\text { Prevalence by each } \\
\text { test (\%) }\end{array}$ & $\begin{array}{c}\text { Number of positives with RBPT negative } \\
\text { with SAT but positive with ELISA }\end{array}$ \\
\hline RBPT & 177 & 21 & 156 & 11.86 & 0 \\
SAT & 177 & 9 & 168 & 5.09 & 0 \\
i-ELISA & 177 & 8 & 169 & 4.52 & 1 \\
\hline
\end{tabular}

i-ELISA: Indirect enzyme-linked immunosorbent assay, SAT: Serum agglutination test, RBPT: Rose bengal plate test

Table-4: The degree of agreement between RBPT, SAT, and i-ELISA tests for human samples.

\begin{tabular}{|c|c|c|c|c|}
\hline Tests & Kappa value & p value & CI (\%) & Degree of agreement \\
\hline RBPT and SAT & 0.57 & $<0.001$ & 95 & Moderate agreement \\
\hline RBPT and i-ELISA & 0.52 & $<0.001$ & 95 & Moderate agreement \\
\hline SAT and i-ELISA & 0.82 & $<0.001$ & 95 & Substantial agreement \\
\hline RBPT, SAT, and i-ELISA & 0.51 & $<0.001$ & 95 & Moderate agreement \\
\hline
\end{tabular}

i-ELISA: Indirect enzyme-linked immunosorbent assay, SAT: Serum agglutination test, RBPT: Rose bengal plate test, CI: Confidence interval 
Table-5: Sensitivity and specificity of RBPT and SAT by comparing with i-ELISA as a gold standard for detection of brucellosis antibodies in goat and human samples.

\begin{tabular}{llcc}
\hline Sample sera & Serological test & Sensitivity & Specificity \\
\hline Goat & RBPT & 100 & 91 \\
\multirow{3}{*}{ Human } & SAT & 100 & 99 \\
& RBPT & 100 & 92 \\
& SAT & 88 & 99 \\
\hline
\end{tabular}

i-ELISA: Indirect enzyme-linked immunosorbent assay, SAT: Serum agglutination test, RBPT: Rose bengal plate test, CI: Confidence interval

smooth Brucella spp., Escherichia coli O116:H21, O157:H7, Francisella tularensis, Salmonella serotypes of Kauffman-White group N, Pseudomonas maltophilia, and Vibrio cholera [13]. The results of SAT and i-ELISA had a substantial degree of agreement for both human and animal samples with kappa value of 0.82 and $p<0.001$ which was statistically significant for both human and animal samples. However, these results are not in agreement with the results observed by Mantur where i-ELISA had the highest seroprevalence compared to SAT and RBPT [14], but the results are in agreement to the results observed by Rahman where RBPT had the highest seroprevalence SAT, and i-ELISA had very close range seroprevalence [15].

In general, there was a moderate degree of agreement between all the three tests in both human and animal with kappa value of 0.57 in animals and 0.51 in humans $95 \%$ confidence interval and $\mathrm{p}<0.001$ which was statistically significant. This was similar to the findings of Elshemey and AbdElrahman [16].

When compared with i-ELISA as a gold standard, RBPT had the highest sensitivity in both goat and human samples while SAT had a higher specificity but lower sensitivity of 88 compared to RBPT of $100 \%$ sensitivity, these results are similar to those obtained by Ahmed et al. [17] but differ with those obtained by Ghodasara et al. [12] which hard i-ELISA with the highest sensitivity and seroprevalence.

The study involved pre-collected samples for other studies and samples suspected for brucellosis that was submitted to the central diagnostic laboratory for brucellosis detection. This might have influenced the results as the sample collection, transport and storage criteria were not clear. A study involving animal and human samples from selected districts in Uganda preferably Serere where most of the samples came from would be appropriate as it will have a clear picture of the relationship between seroprevalence in animals and humans.

\section{Conclusion}

In this study, RBPT detected the highest seroprevalence of brucellosis with the highest sensitivity compared to other serological tests. Thus, RBPT could be used as a suitable screening test for diagnosis of brucellosis in goat and humans. All i-ELISA positive samples were tested positive by RBPT indicating that i-ELISA test could be used for confirmatory diagnosis of brucellosis. All RBT positive samples were tested positive with SAT indicating that SAT could also be used as a confirmatory test.

\section{Authors' Contributions}

IPA: Provided part of laboratory supplies, developed the concept, participated in proposal/protocol writing, laboratory work, and manuscript writing. DN: Provided the laboratory supplies, participated in proposal writing, and laboratory work. CDK: Participated in proposal writing, presentation of the research methods to the research and ethics committee, and manuscript writing. SK: Participated in the laboratory work and manuscript writing. KI: Participated in concept development, presentation of the research methods to the research and ethics committee, and manuscript writing. GT: Participated in protocol/proposal writing, obtained samples from the archives, and manuscript writing. BN: Participated in laboratory work and manuscript writing. All authors read and approved the final manuscript.

\section{Acknowledgments}

We acknowledge, Makerere University, College of Veterinary Medicine Animal Resources and Biosecurity and Central Diagnostic Laboratory lab stuff for the support, facilities and conducive environment provided. We acknowledge Mr. Ndoboli Dickson for providing the kits and the reagents.

\section{Competing Interests} interests.

The authors declare that they have no competing

\section{References}

1. Kakooza S, Tumwebaze M, Nabatta E, Byaruhanga J, Tayebwa DS, Wampande E. Risk factors and co-existence of infectious causes of reproductive failures in selected Uganda cattle and goats: A Brucella spps-Toxoplasma gondii study. Open Access Libr J 2018;5:1.

2. Bamaiyi PH. Prevalence and risk factors of brucellosis in man and domestic animals : A review. Int J One Heal 2016;2:29-34.

3. Seleem MN, Boyle SM, Sriranganathan N. Brucellosis: A re-emerging zoonosis. Vet Microbiol 2010; 140:392-8.

4. Makita $\mathrm{K}$, Fèvre EM, Waiswa $\mathrm{C}$, Eisler MC, Thrusfield M, Welburn SC. Herd prevalence of bovine brucellosis and analysis of risk factors in cattle in urban and peri-urban areas of the Kampala economic zone, Uganda. BMC Vet Res 2011; 7:60.

5. Gall D, Nielsen K. Serological diagnosis of bovine brucellosis: A review of test performance and cost comparison. Rev Sci Tech 2004; 23:989-1002.

6. Mangalgi S, Sajjan A, Mohite S, Kakade S. Human brucellosis: Still an unfamiliar and misdiagnosed disease in India. J Krishna Inst Med Sci Univ 2015; 4:59-65.

7. Kumar A. Brucellosis: Need for public health intervention in rural India. Prilozi 2010; 31:219-31.

8. Mangen MJ, Otte J, Pfeiffer D, Chilonda P. Bovine Brucellosis in Sub-Saharan Africa: Estimation of Seroprevalence and Impact on Meat and Milk Off-take Potential. 
FAO Livest Policy Discuss Pap No. 8, No. 8, p. 58; 2002.

9. Alton GG, Jones LM, Pietz DE. Laboratory techniques in brucellosis. Monogr Ser World Health Organ 1975; 55:1-63.

10. Omer MK, Skjerve E, MacMillan AP, Woldehiwet Z. Comparison of three serological tests in the diagnosis of Brucella infection in unvaccinated cattle in Eritrea. Prev Vet Med 2001;48:215-22.

11. Din AM, Khan SA, Ahmad I, Rind R, Hussain T, Shahid M, et al. A study on the seroprevalence of brucellosis in human and goat populations of district bhimber, Azad Jammu and Kashmir. J Anim Plant Sci 2013; 23:113-8.

12. Ghodasara SN, Roy A, Bhanderi BB. Comparison of rose bengal plate agglutination, standard tube agglutination and indirect ELISA tests for detection of Brucella antibodies in cows and buffaloes. Vet World 2010; 3:61-4.

13. Ingold T. Review article. J R Anthropol Inst 2014; 4:771-3.
14. Mantur B, Parande A, Amarnath S, Patil G, Walvekar R, Desai A, et al. ELISA versus conventional methods of diagnosing endemic brucellosis. Am J Trop Med Hyg 2010; 83:314-8

15. Rahman AK. Comparative evaluation of commercial serodiagnostic tests for the seroprevalence study of brucellosis in stray dogs in Bangladesh. Bangladesh J Vet Med 2011; 9:79-83.

16. Elshemey T, Abd-Elrahman A. Evaluation of a rapid immunochromatographic test for detection of Brucella abortus antibodies in Egyptian cattle sera and milk. Alexandria J Vet Sci 2014; 40:24-8.

17. Ahmed M, Islam MA, Khatun MM, Baek BK. Evaluation of four serological tests for the detection of brucellosis in goats and cattle under the field condition of Bangladesh. Asian J Biol Sci 2011; 4:477-82.

$* * * * * * * *$ 